\title{
Orthostatic Hypotension and Concurrent Autonomic Dysfunction: A Novel Complication of Lung Transplantation
}

\author{
Deepika Razia (D), ${ }^{1,2}$ Sofya Tokman ${ }^{D}$, 1,2 Sharjeel Israr, ${ }^{1,2}$ Hesham Mohamed, ${ }^{1,2}$ \\ Hesham Abdelrazek, ${ }^{1,2}$ Bhuvin Buddhdev, ${ }^{1,2}$ Ashwini Arjuna $\mathbb{D}^{1,},{ }^{1,2}$ Kendra McAnally, ${ }^{1,2}$ \\ Samad Hashimi, ${ }^{1,2}$ Michael A. Smith, ${ }^{1,2}$ Ross M. Bremner $\mathbb{D}^{1,2}$ Rajat Walia $\mathbb{D}^{1,2}$ \\ and Ashraf Omar $\mathbb{D}^{1,2}$
}

${ }^{1}$ Norton Thoracic Institute, St. Joseph's Hospital and Medical Center, Phoenix, AZ, USA

${ }^{2}$ Creighton University School of Medicine, Phoenix Regional Campus, Phoenix, AZ, USA

Correspondence should be addressed to Ashraf Omar; ashraf.omar@dignityhealth.org

Received 1 July 2021; Revised 6 January 2022; Accepted 25 January 2022; Published 3 March 2022

Academic Editor: Amar Singh

Copyright (C) 2022 Deepika Razia et al. This is an open access article distributed under the Creative Commons Attribution License, which permits unrestricted use, distribution, and reproduction in any medium, provided the original work is properly cited.

Background. Persistent orthostatic hypotension $(\mathrm{OH})$ is a lesser-known complication of lung transplantation (LTx). In this retrospective case series, we describe the clinical manifestations, complications, and treatment of persistent $\mathrm{OH}$ in $13 \mathrm{LTx}$ recipients. Methods. We identified LTx recipients who underwent transplantation between March 1, 2018, and March 31, 2020, with persistent symptomatic $\mathrm{OH}$ and retrospectively queried the records for clinical information. Results. Thirteen patients were included in the analysis, 9 (69\%) had underlying pulmonary fibrosis, and 12 (92\%) were male. The median age, height, and body mass index at LTx were 68 years, 70 inches, and $27 \mathrm{~kg} / \mathrm{m}^{2}$, respectively. Six (46\%) patients were deceased at the time of chart abstraction with a median (IQR) posttransplant survival of 12.6 months $(6,21)$; the 7 remaining living patients were a median of 19.6 months $(18,32)$ posttransplant. Signs and symptoms of $\mathrm{OH}$ developed a median of $60(7,75)$ days after transplant. Patients were treated with pharmacological agents and underwent extensive physical therapy. Most patients required inpatient rehabilitation $(n=10,77 \%)$, and patients commonly developed comorbid conditions including weight loss, renal insufficiency with eGFR $<50(n=13,100 \%)$, gastroparesis $(n=7,54 \%)$, and tachycardia-bradycardia syndrome $(n=2,15 \%)$. Falls were common $(n=10,77 \%)$. The incidence of $\mathrm{OH}$ in LTx recipients at our center during the study period was 5.6\% (13/234). Conclusions. Persistent $\mathrm{OH}$ is a lesser-known complication of LTx that impacts posttransplant rehabilitation and may lead to comorbidities and shortened survival. In addition, most LTx recipients with $\mathrm{OH}$ at our center were tall, thin men with underlying pulmonary fibrosis, which may offer an opportunity to instate pretransplant $\mathrm{OH}$ screening of at-risk patients.

\section{Introduction}

Lung transplantation (LTx) is a lifesaving procedure for patients with end-stage lung disease; however, it is associated with several complications that impact both quality of life and longevity. Persistent orthostatic hypotension $(\mathrm{OH})$ is an unusual complication of LTx with significant clinical implications. $\mathrm{OH}$ is defined as a reduction of systolic blood pressure (SBP) of at least $20 \mathrm{mmHg}$ or diastolic blood pressure $(\mathrm{DBP})$ of at least $10 \mathrm{mmHg}$ within 3 minutes of standing and/or an increase in heart rate $\geq 20$ beats/minute but not exceeding the upper limit of normal $[1,2]$. OH may result from an inadequate response to postural changes in blood pressure $[1,2]$ and may lead to cerebral hypoperfusion with resultant dizziness, visual disturbances, and syncope; muscular hypoperfusion with resultant myalgia; and renal hypoperfusion with resultant oliguria. It is also associated with nonspecific symptoms such as weakness, lethargy, fatigue, and falls [3].

$\mathrm{OH}$ may also be part of a clinical syndrome of autonomic dysfunction (AD), which is characterized by cardiac, gastrointestinal, and genitourinary disturbances. AD may present as arrhythmias (tachycardia-bradycardia syndrome and atrial fibrillation), ileus, Ogilvie syndrome, 
gastroparesis, urinary complaints (retention, hesitancy, dysuria, and incontinence), and bowel complaints (diarrhea and constipation) [3].

Physical therapy and rehabilitation are key components of LTx recovery [4]. Despite near-normal lung function, exercise intolerance and reduction in quality of life (QOL) often persist years after transplantation [5-14]. Muscle dysfunction, inactivity, deconditioning, and nutritional depletion can affect exercise capacity before LTx, and an extended hospital and intensive care unit (ICU) stay may impact lung recipients' recovery in terms of exercise tolerance and QOL [15]. Physical rehabilitation after transplantation can improve exercise capacity and reduce the risk of osteoporosis and muscle dysfunction [16]. OH and other symptoms of $\mathrm{AD}$ impact a patient's ability to participate in rehabilitation, hence limiting a successful recovery after LTx.

Persistent $\mathrm{OH}$, alone or in conjunction with $\mathrm{AD}$, can lead to significant comorbidities after LTx and can also impair posttransplant rehabilitation. In a pilot sample of 30 consecutive stable LTx recipients from the University of Zurich, the prevalence of undiagnosed $\mathrm{OH}$ was $30 \%(n=9), 3$ of these patients were symptomatic, and another 3 had supine hypertension [17]. Although markers of $\mathrm{AD}$ as prognostic indicators have been studied in chronic pulmonary diseases and LTx recipients [18-22], the morbidity associated with $\mathrm{OH}$ and $\mathrm{AD}$ has not been described. In this retrospective case series, we describe the clinical course of 13 LTx recipients with persistent $\mathrm{OH}$.

\section{Methods}

Institutional review board approval with a waiver of patient consent was obtained for this study (PHXU-21-500-137-7318, dated March 31, 2021). Patients who underwent LTx between March 1, 2018, and March 31, 2020, at Norton Thoracic Institute, Phoenix, Arizona, with persistent symptomatic $\mathrm{OH}$ any time after LTx were included. $\mathrm{OH}$ was defined as a reduction of SBP of at least $20 \mathrm{mmHg}$ or a reduction of DBP of at least $10 \mathrm{mmHg}$ within 3 minutes of standing and/or an increase in heart rate $\geq 20$ beats/minute but not exceeding the upper limit of normal $[1,2]$. Of note, only LTx recipients with ongoing postural dizziness and falls at home, clinic, or hospital were screened and diagnosed with persistent $\mathrm{OH}$. A diagnosis of persistent $\mathrm{OH}$ triggered the evaluation of symptoms of $\mathrm{AD}$ [3]. Demographic characteristics, medical history, and clinical outcomes were reviewed for all included patients. Gastroparesis was noted on scintigraphy studies before and after LTx. The six-minute walk distance closest to the time of LTx was reported.

2.1. Immunosuppression. Induction therapy included a high-dose corticosteroid (methylprednisolone) before perfusion of each lung allograft and antilymphocyte therapy with basiliximab, rituximab, or antithymocyte globulin (ATG). At our center, the vast majority of patients are induced with basiliximab, with the exception of highly sensitized patients and patients undergoing retransplant who developed allograft failure as a result of recurrent or persistent acute cellular or antibody-mediated rejection. Highly sensitized patients typically receive rituximab or ATG at the time of transplant; whereas, patients undergoing retransplant commonly receive ATG. The maintenance immunosuppressive regime was uniform across the study period and consisted of a corticosteroid (prednisone), an antiproliferative agent (mycophenolate mofetil or mycophenolic acid), and a calcineurin inhibitor ((CNI), tacrolimus or cyclosporine). A selective $\mathrm{T}$ cell costimulation blocker (belatacept) was used to reduce CNI-induced nephrotoxicity in patients with progressive and advanced chronic kidney disease.

2.2. Statistics. Descriptive statistics were used. Median and interquartile range (IQR) were reported for continuous variables. Frequencies and percentages were reported for categorical variables. The Kaplan-Meier method and logrank test were used for comparison of survival estimates between included subjects and a control group of remaining subjects who underwent LTx during the study period. Analyses were performed with Stata Statistical Software, Release 13 (Stata Corp College Station, TX).

\section{Results}

During the study period, 234 patients (137 males) with a median (IQR) age of 67.2 years $(59.2,70.5)$ underwent LTx at our center.

3.1. Pre-LTx Clinical Features. Thirteen patients with post$\mathrm{LTx} \mathrm{OH}$ were included in this series. The incidence of $\mathrm{OH}$ in LTx recipients at our center during the study period was $5.6 \%(13 / 234)$. The median age of the study cohort at the time of LTx was $68(66,71)$ years, and 92.3\% $(n=12)$ were male. Median height and body mass index (BMI) at LTx were $70(68,72.5)$ inches and $26.6(22.8,28.9) \mathrm{kg} / \mathrm{m}^{2}$, respectively; however, 2 of the patients had a BMI $<18.5 \mathrm{~kg} / \mathrm{m}^{2}$. Most patients had idiopathic pulmonary fibrosis (69.2\%; $n=9$ ), and the remaining patients had a either chronic obstructive pulmonary disease $(23.1 \% ; n=3)$ or combined pulmonary fibrosis and emphysema $(7.7 \% ; n=1)$ (Table 1$)$.

All patients were functionally impaired prior to LTx; New York Heart Association Grade III functional class was observed in $84.6 \%(n=11)$ of patients and grade IV was observed in $15.4 \%(n=2)$. Median oxygen requirement at rest was $3(3,6.5)$ liters per minute, and $15.4 \%(n=2)$ of patients required continuous positive airway pressure for sleep apnea. The median pre-LTx forced expiratory volume in one second (FEV1) was $45 \%(23,59)$, and the median forced vital capacity (FVC) was 49\% (40, 61); the lowest FEV 1 was $16 \%$ of predicted, and the lowest FVC was $18 \%$ of predicted. Pre-LTx hypercapnia with nonobstructive pathology was seen in $30.8 \%(n=4)$ of patients. None of the patients had valvular heart disease.

Although systemic hypertension was common $(61.5 \%$, $n=8$ ), only 1 patient carried a pre-LTx diagnosis of $\mathrm{OH}$ and was treated with midodrine. Pre-LTx arrhythmias were common $(30.8 \%, n=4)$ with atrial fibrillation (AFib) in 
TABLE 1: Baseline characteristics, pretransplant clinical features, and outcomes in the study cohort.

\begin{tabular}{|c|c|}
\hline Variables & $\begin{array}{c}\text { Study cohort, } \\
\quad n=13\end{array}$ \\
\hline Age, years $^{\dagger}$ & $68(66,71)$ \\
\hline Gender, male & $12,92.3$ \\
\hline Height, inches $^{\dagger}$ & $70(68,72.5)$ \\
\hline Body mass index, $\mathrm{kg} / \mathrm{m}^{2 \dagger}$ & $26.6(22.8,28.9)$ \\
\hline Lung transplant, bilateral & $12,92.3$ \\
\hline \multicolumn{2}{|l|}{ Underlying diagnosis } \\
\hline IPF & $9,69.2$ \\
\hline COPD & $3,23.1$ \\
\hline CPFE & $1,7.7$ \\
\hline \multicolumn{2}{|l|}{ Pre-LTx clinical features } \\
\hline $\mathrm{LAS}^{\dagger}$ & $47.5(35.4,54.7)$ \\
\hline Creatinine, $\mathrm{mg} / \mathrm{dL}^{\dagger}$ & $0.8(0.8,1.2)$ \\
\hline Distance walked in six minutes, meters ${ }^{\dagger}$ & $346(273,393)$ \\
\hline Right atrial pressure, $\mathrm{mmHg}^{\dagger}$ & $7.5(4,12.5)$ \\
\hline Mean pulmonary artery pressure, $\mathrm{mmHg}^{\dagger}$ & $22(20,33)$ \\
\hline Pulmonary capillary wedge pressure, $\mathrm{mmHg}^{\dagger}$ & $15(6,19)$ \\
\hline Cardiac index & $3.3(2.9,3.7)$ \\
\hline Known orthostatic hypotension & $1,7.7$ \\
\hline Arrhythmia & $4,30.8$ \\
\hline Preexisting peripheral neuropathy & $3,23.1$ \\
\hline Preexisting gastroparesis & $2,15.4$ \\
\hline \multicolumn{2}{|l|}{ Immunosuppression } \\
\hline Induction: basiliximab & $10,76.9$ \\
\hline Induction: rituximab/IVIG & $2,15.4$ \\
\hline Induction: ATG & $1,7.7$ \\
\hline Mycophenolate mofetil, tacrolimus, steroids & 13,100 \\
\hline Cyclosporin & $1,7.7$ \\
\hline Belatacept & $1,7.7$ \\
\hline \multicolumn{2}{|l|}{ Post-LTx clinical features } \\
\hline Primary graft dysfunction grade 3 & $4,30.8$ \\
\hline Time to endotracheal extubation, days ${ }^{\dagger}$ & $4(1,6)$ \\
\hline Atrial fibrillation in first postoperative week & $9,69.2$ \\
\hline Decrease in SBP supine to standing ${ }^{\dagger}$ & $56(38,66)$ \\
\hline Decrease in DBP supine to standing ${ }^{\dagger}$ & $25(10.5,40)$ \\
\hline Supine hypertension & $10,76.9$ \\
\hline Discharged to home & $3,23.1$ \\
\hline Inpatient rehabilitation after LTx & $10,76.9$ \\
\hline Readmissions for inpatient rehabilitations & $3,23.1$ \\
\hline Number of readmissions in first year ${ }^{\dagger}$ & $2(1,3)$ \\
\hline Length of stay for readmissions, days ${ }^{\dagger}$ & $18(13,29)$ \\
\hline Deaths & $6,46.2$ \\
\hline $1^{\text {st }}$ year & $3,23.1$ \\
\hline $2^{\text {nd }}$ year & $2,15.4$ \\
\hline $3^{\text {rd }}$ year & $1,7.7$ \\
\hline
\end{tabular}

Data expressed as numbers, percentages unless specified otherwise; ${ }^{\dagger}$ data expressed as median (interquartile range). IPF, idiopathic pulmonary fibrosis; COPD, chronic obstructive pulmonary disease; CPFE, combined pulmonary fibrosis and emphysema; LTx, lung transplant; LAS, lung allocation score; IVIG, intravenous immunoglobulin; ATG, rabbit antithymocyte globulin; SBP, systolic blood pressure; DBP, diastolic blood pressure.

$23.1 \%(n=3)$ of patients and left bundle branch block in $7.7 \%(n=1)$. Pre-LTx diabetes was present in 30.8\% $(n=4)$ of patients; $23.1 \%(n=3)$ had peripheral neuropathy, and 1 patient was insulin-dependent. Gastroparesis was present in $15.4 \% \quad(n=2)$ of patients, one of whom was diabetic.
Osteopenia or osteoporosis was present in 30.8\% $(n=4)$ of patients, and $15.4 \%(n=2)$ had chronic kidney disease (stage III).

3.2. Peri-LTx Clinical Features. Most patients underwent bilateral LTx $(92.3 \%, n=12)$. Cardiopulmonary bypass was used in 1 patient and extracorporeal membrane oxygenation in 2 patients. Concomitant left atrial appendage clipping was performed in $15.4 \%(n=2)$ of patients. A thoracic epidural catheter was placed before induction of anesthesia uneventfully in $76.9 \%(n=10)$ of patients. Intraoperative arrhythmias were seen in $38.5 \%(n=5)$ of patients: AFib needing cardioversion in $23.1 \%(n=3)$, supraventricular tachycardia in $7.7 \%(n=1)$, and ventricular tachycardia in $7.7 \%(n=1)$. Intraoperative right ventricular function and pulmonary vein velocity were normal in all patients. Median blood loss was $1(0.6,2.3)$ liter, and blood products were transfused in $46.2 \%(n=6)$ of patients.

Four patients $(30.8 \%)$ developed grade 3 primary graft dysfunction (PGD), and the median time to extubation of these patients was 6 days $(4,7) ; 1$ of these patients had a tracheostomy for prolonged mechanical ventilation. The median time to extubation was 4 days $(1,6)$ in the entire cohort.

Most patients developed cardiac arrhythmia in the immediate postoperative period. Nine patients (69.2\%) developed AFib with rapid ventricular response within the first postoperative week, which was managed with antiarrhythmic agents and cardioversion. A dual-chamber pacemaker was placed in 1 patient 2 weeks after LTx, and radiofrequency ablation was performed in 1 patient 5 months after LTx.

Protracted post-LTx hospitalization was common. All patients $(n=13)$ required a $>1$-week stay in the ICU; the median duration of hospitalization was 40 days $(23,47)$, and $76.9 \%(n=10)$ of patients were discharged to an inpatient acute rehabilitation facility. Readmission within the first year of LTx was common; $84.6 \%(n=11)$ had $\geq 1$ readmission within the first year, with a median number of 2 readmissions. The median duration of readmission hospital stay was 18 days $(13,29)$, and 3 patients required readmission to an inpatient acute rehab facility.

3.3. Post-LTx Clinical Features. OH was typically diagnosed early in the post-LTx course with 12 patients (92.3\%) diagnosed within 3 months of transplant. The median decrease in SBP and DBP from supine to standing position was 56 (38, 66) and $25(10.5,40) \mathrm{mmHg}$, respectively. Patients commonly reported dizziness $(84.6 \%, n=11)$, limited ambulation $(76.9 \%, n=10)$, falls $(76.9 \%, n=10)$, and muscle weakness $(69.3 \%, n=9)$. Patients also commonly developed comorbid conditions including weight loss (median decrease in BMI compared to pre-LTx BMI was $2.7 \mathrm{~kg} / \mathrm{m}^{2}$ ) and renal insufficiency with eGFR $<50 \%(n=13,100 \%)$. Fractures sustained during mechanical fall episodes occurred in $46.2 \%$ $(n=6)$ of patients, and $38.5 \%(n=5)$ required orthopedic 
surgery. Deconditioning and failure to thrive were present in all patients $(n=13)$ with decubitus ulcers in $15.4 \%(n=2)$. Clinical depression was treated in $30.8 \%(n=4)$ of patients, and 1 patient had generalized anxiety related to fear of syncope.

All patients with $\mathrm{OH}(n=13)$ also had additional symptoms of $\mathrm{AD}$ that could not be attributed to $\mathrm{OH}$ alone. The presentation of $\mathrm{AD}$ included urinary complaints (retention, dysuria, incontinence, and hesitancy; $61.5 \%, n=8$ ), bowel dysregulation (diarrhea $(61.5 \%, n=8)$, gastroparesis $(53.8 \%, n=7)$, ileus $(100 \%, n=13)$, Ogilvie syndrome with cecal dilation $>10 \mathrm{~cm}(15.4 \%, n=2)$, labile blood glucose levels requiring frequent insulin dose adjustments $(61.5 \%$, $n=8)$, tachycardia-bradycardia syndrome $(15.4 \%, n=2)$, sweating paroxysms with new-onset peripheral neuropathy $(7.7 \%, n=1)$, and dry mouth with oral mucosal fragility $(7.7 \%, n=1)$ (Table 2; Figure 1).

Testosterone levels were measured in 8 male patients, 7 of whom had hypogonadism with a median level of total testosterone, bioavailable testosterone, and free testosterone of $151 \mathrm{ng} / \mathrm{dL}, 59 \mathrm{ng} / \mathrm{dL}$, and $25 \mathrm{pg} / \mathrm{mL}$, respectively. Six (46\%) of the patients were deceased at the time of chart abstraction with a median posttransplant survival of 12.6 months $(6,21) ; 7$ living patients were a median of 19.6 months $(18,32)$ posttransplant. Follow-up was available for a median of $17.7(14.2,29.6)$ months, and median survival was $17.4(9.2,23.2)$ months.

Pharmacologic and mechanical agents were used to treat $\mathrm{OH}$. Commonly used medications included midodrine $(100 \%$, $n=13)$, fludrocortisone $(53.8 \%, n=7)$, and droxidopa $(53.8 \%$, $n=7$; Table 3 ). These medications were used in an escalating fashion with midodrine started first, fludrocortisone added second, and droxidopa added third for treatment of refractory patients who could obtain insurance approval for this medication. Drug side effects were common and included supine hypertension $(76.9 \%, n=10)$, fluid retention, and hyponatremia, with the latter two particularly common among patients treated with fludrocortisone. In an attempt to reduce tacrolimus-induced $\mathrm{OH}, 1$ patient was transitioned to a cyclosporinebased regimen and 1 patient was treated with belatacept, which allowed us to target lower tacrolimus troughs. Compression socks, TED hose, and abdominal binders were used in all patients. A port for outpatient administration of intravenous fluids on a biweekly basis was placed in 1 patient. Despite $\geq 2$ treatment strategies, orthostatic signs were challenging to treat in $53.8 \%(n=7)$ of patients. Four patients were on triple therapy (midodrine, fludrocortisone, and droxidopa); none of these 4 patients were alive at the end of the study period. Of those who were alive $(n=7), \mathrm{OH}$ was self-limiting in 1 patient and recalcitrant to therapy in 6 patients who still require some form of treatment.

Last, the Kaplan-Meier survival analysis between the study group and the remaining LTx recipients during the study period was conducted. The probability of survival at 3 years was significantly lower in the study group than in the control group ( $46.2 \%$ vs. $74.8 \%, p=0.008$, Figure 2$)$. The median (IQR) survival of the study group and controls was 25.1 (16.5, 33.7) months and 37.8 (32.5, 43.1) months, respectively.

\section{Discussion}

We identified 13 LTx recipients at our center with OH; 100\% $(n=13)$ had additional symptoms of $\mathrm{AD}$ with resultant significant morbidity and mortality. The etiology of $\mathrm{OH}$ and $\mathrm{AD}$ after LTx remains unclear; however, several hypotheses have been proposed. One hypothesis focuses on vagus nerve injury. The right and left vagus nerves contribute to the cardiac, pulmonary, and esophageal plexuses and enter the diaphragm through the esophageal hiatus as the posterior and anterior vagal trunks. In light of its course through the thoracic cavity, it may be subject to mechanical, ischemic, and hypothermic injury at the time of transplant, and the manifestations of the injury may vary depending on which branch was affected.

Although intraoperative vagus nerve injury may explain $\mathrm{OH}$ and $\mathrm{AD}$ in the early post-LTx course, additional factors need to be considered since 7.7\% $(n=1)$ developed $\mathrm{OH}$ and AD 17 months after surgery. Notably, the patient who developed $\mathrm{OH}$ and $\mathrm{AD}>6$ months after transplant was $>65$ years old. $\mathrm{OH}$ is a well-described complication of aging and senescence [23, 24]. An association between age-related frailty and degenerative dysautonomia has been demonstrated in a community-dwelling population of 65-101 years old [24]. In frail older patients, baroreflex sensitivity, $\alpha-1$ adrenergic vasoconstrictor response to sympathetic stimuli, and parasympathetic activity may be altered. In addition, renal salt and water conservation, comorbidity burden, and impaired thirst response to dehydration may be associated with poor homeostasis to orthostatic stress [25]. Furthermore, sarcopenia, neuromuscular frailty, or critical illness myopathy potentially compromise venous return and result in $\mathrm{OH}$.

Additionally, post-LTx medications may contribute to the development of $\mathrm{OH}$. For example, CNIs, the backbone of posttransplant immunosuppressive therapy in solid organ transplant recipients, have been reported to cause peripheral neuropathy. Bhagavati et al. [26] reported 2 patients who developed chronic sensorimotor polyneuropathy after tacrolimus use following renal transplantation. One patient had an unusual presentation with bilateral facial and extremity weakness and a relapsing course. The other patient presented with focal sensory symptoms in one hand. Electrophysiological studies confirmed widespread, predominantly demyelinating or axonal polyneuropathy. Although highly speculative, it is theoretically possible that CNIs have a similar impact on the autonomic nervous system, thereby leading to $\mathrm{OH}$ and $\mathrm{AD}$.

Last and notably, the vast majority of LTx recipients with $\mathrm{OH}$ were male, raising the possibility that sex hormones play a role in this condition. Testosterone deficiency and hypogonadism have been described after solid organ transplantation. Fleischer et al. [27] measured serum testosterone, estradiol, sex hormone-binding globulin, and gonadotropin levels for the first two years after transplantation in a cohort of 108 heart transplant recipients. Total and free testosterone levels were lowest during the first month $(257 \pm 131$ and $6.2 \pm 3 \mathrm{ng} / \mathrm{dl}$, respectively) and normalized by 2 months. Gonadotropins were low in the majority, suggesting 
TABLE 2: Manifestations of autonomic dysfunction in individual patients by patient ID.

\begin{tabular}{|c|c|c|c|c|c|c|c|c|c|c|c|}
\hline $\begin{array}{l}\text { Patient } \\
\text { ID }\end{array}$ & $\mathrm{OH}$ & Dizziness & $\begin{array}{c}\text { AFib/ } \\
\text { tachycardia- } \\
\text { bradycardia }\end{array}$ & Myopathy & $\begin{array}{c}\text { Ogilvie } \\
\text { syndrome }\end{array}$ & $\begin{array}{l}\text { Labile } \\
\text { BS }\end{array}$ & $\begin{array}{c}\text { Urinary } \\
\text { complaints }\end{array}$ & $\begin{array}{c}\text { Bowel } \\
\text { dysregulation }\end{array}$ & DGE & Falls & Hypogonadism \\
\hline 1 & + & + & + & + & & + & + & + & + & + & \\
\hline 2 & + & + & + & + & & & & & & + & + \\
\hline 3 & + & + & + & + & + & & + & & + & + & + \\
\hline 4 & + & + & & & & & + & + & & & + \\
\hline 5 & + & + & & + & + & + & + & & + & + & + \\
\hline 6 & + & + & + & + & & + & & & + & & \\
\hline 7 & + & + & + & & & + & + & & & + & \\
\hline 8 & + & + & + & & & + & & + & & + & \\
\hline 9 & + & + & + & + & & + & & + & & + & \\
\hline 10 & + & + & + & + & & + & + & + & + & & + \\
\hline 11 & + & & + & + & & & & + & + & + & + \\
\hline 12 & + & & + & & & & + & + & & + & + \\
\hline 13 & + & + & & + & & + & + & + & + & + & \\
\hline
\end{tabular}

+ , present. $\mathrm{OH}$, orthostatic hypotension; AFib, atrial fibrillation; BS, blood sugar; DGE, delayed gastric emptying.

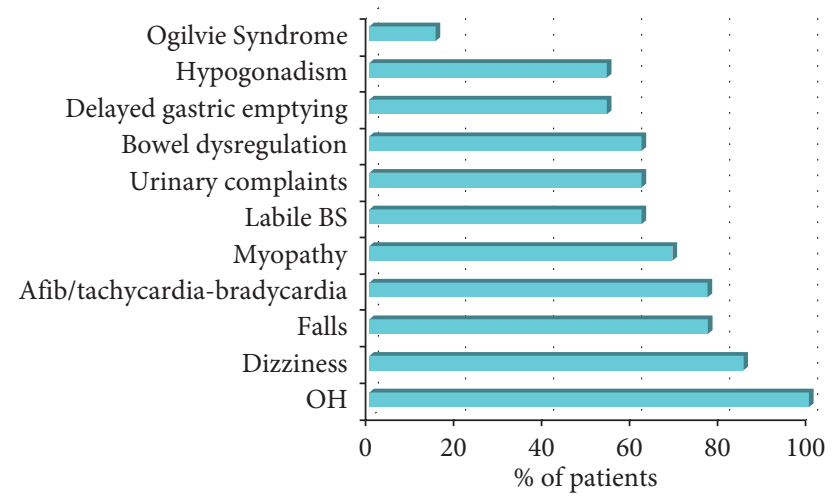

FIGURE 1: Prevalence and presentation of autonomic dysfunction in lung transplant recipients. OH, orthostatic hypotension; BS, blood sugar.

TABle 3: Treatment strategies for orthostatic hypotension in individual patients by patient ID.

\begin{tabular}{|c|c|c|c|c|c|c|c|}
\hline $\begin{array}{l}\text { Patient } \\
\text { ID }\end{array}$ & Midodrine & Fludrocortisone & Droxidopa & Desmopressin & $\begin{array}{l}\text { Pyridostigmine/ } \\
\text { neostigmine }\end{array}$ & $\begin{array}{l}\text { Mechanical treatment } \\
\text { strategies (compression socks/ } \\
\text { TED hose/abdominal binders) }\end{array}$ & Physiotherapy \\
\hline 1 & + & + & & & & + & + \\
\hline 2 & + & + & + & & & + & + \\
\hline 3 & + & + & + & + & + & + & + \\
\hline 4 & + & & & & & & + \\
\hline 5 & + & & + & & & + & + \\
\hline 6 & + & + & & & & + & + \\
\hline 7 & + & & + & & & + & + \\
\hline 8 & + & & & & & & + \\
\hline 9 & + & + & + & + & + & + & + \\
\hline 10 & + & + & + & & & + & + \\
\hline 11 & + & & & & & + & + \\
\hline 12 & + & + & & & & + & + \\
\hline 13 & + & & + & & & + & + \\
\hline
\end{tabular}

+ , yes.

hypothalamic-pituitary-gonadal axis suppression. Low total testosterone persisted in $14 \%$ of patients at 1 year and $18 \%$ at 2 years. Prednisone was the major predictor of serum testosterone. Although there is no obvious mechanism driving both hypogonadism and $\mathrm{OH}$ and $\mathrm{AD}$, it is possible that hypogonadism contributes to frailty in male LTx recipients, making blood pressure fluctuations less tolerable.

Although the etiology of $\mathrm{OH}$ and AD post-LTx remains unknown, it clearly has a profound impact on QOL, morbidity, and mortality for the LTx recipients that developed 


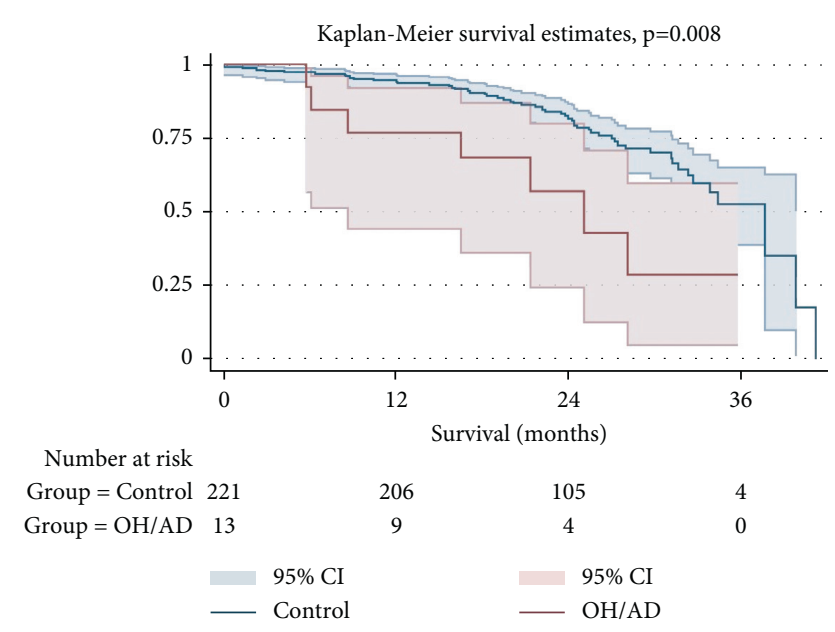

Figure 2: Kaplan-Meier survival analysis stratified by subjects with posttransplant orthostatic hypotension and/or autonomic dysregulation and a control group of remaining subjects transplanted during the study period. $\mathrm{OH}$, orthostatic hypotension; $\mathrm{AD}$, autonomic dysregulation; CI, confidence interval.

this syndrome. Patients with $\mathrm{OH}$ were commonly dizzy, weak, and frail and had difficulty with physical rehabilitation due to these symptoms. They suffered from renal failure and recurrent falls, some of which required orthopedic intervention. $\mathrm{OH}$ was commonly accompanied by additional symptoms of $\mathrm{AD}$, including urinary complaints (retention, dysuria, incontinence, and hesitancy), diarrhea, labile blood glucose levels, tachycardia-bradycardia syndrome, gastroparesis, ileus, Ogilvie syndrome with cecal dilation $>10 \mathrm{~cm}$, sweating paroxysms with new-onset peripheral neuropathy, and dry mouth with oral mucosal fragility (Table 2; Figure 1). These symptoms likely exacerbated an already tenuous clinical state and almost certainly contributed to fragility and poor QOL. Similar morbidity associated with $\mathrm{OH}$ has been described in other solid organ transplant recipients. Nygaard et al. [28] described dizziness and poor functional status in heart transplant recipients; Kuten et al. [29] reported multiple readmissions in pancreas transplant recipients; and Khurana et al. [30] and Munjal et al. [31] reported urinary complaints and hypertensive hemorrhagic infarction, accordingly, in pancreas-kidney transplant recipients.

Persistent $\mathrm{OH}$ can be treatment refractory despite the use of both pharmacological and mechanical therapies. Our standard protocol is to rule out adrenal insufficiency and use intravenous fluids, mechanical strategies (such as TED hose and abdominal binders), midodrine, fludrocortisone, and droxidopa on a caseto-case basis, along with outpatient physiotherapy. Escalating doses of medications and polypharmacy may lead to untoward side effects including supine hypertension, edema, and electrolyte disturbances. Mechanical devices such as TED hose may be difficult to put on, particularly by weak patients, and abdominal binders may restrict breathing and food intake. The complications of $\mathrm{AD}$ are also difficult to treat. For example, gastroparesis often required multiple interventions, including endoscopy with pyloric dilation and botulinum toxin injection, and patients are often left malnourished, nauseous, and feeding tube dependent.
Our report has several limitations. First, it is a descriptive, single-center case series with low levels of evidence. Nevertheless, to the best of our knowledge, this is the first series in the LTx literature describing $\mathrm{OH}$ and $\mathrm{AD}$ as possible complications of LTx. Second, we have likely underestimated the incidence of post-LTx $\mathrm{OH}$ and $\mathrm{AD}$ as patients are not routinely screened for these conditions, and therefore, minimally symptomatic patients were not identified. Third, since the clinical presentation of $\mathrm{AD}$ is so variable, it is difficult to differentiate between symptoms attributable to $\mathrm{AD}$ versus those attributable to other etiologies. However, despite these challenges, the syndrome of $\mathrm{OH}$ and $\mathrm{AD}$ is worthy of formal description in the literature as well as ongoing study as it has a profound impact on postLTx morbidity and mortality. In fact, as a result of these observations after LTx, our center has incorporated a formal assessment of orthostasis as part of the standard pre-LTx evaluation. However, further large studies are needed to better define this syndrome and its impact on the clinical outcomes of lung transplant recipients.

\section{Conclusions}

$\mathrm{OH}$ with concurrent $\mathrm{AD}$ is a newly described complication of LTx that leads to significant comorbidities, impairs postLTx rehabilitation, and may be associated with shortened post-LTx survival. The majority of LTx recipients with $\mathrm{OH}$ at our center were tall, thin men with underlying pulmonary fibrosis, which may offer an opportunity to instate pretransplant $\mathrm{OH}$ screening of at-risk patients. Further studies are needed to identify risk factors for $\mathrm{OH}$ and $\mathrm{AD}$ as well as to identify the etiology and pathophysiology of this syndrome.

\section{Abbreviations}

AD: Autonomic dysfunction

AFib: Atrial fibrillation

ATG: Antithymocyte globulin

BMI: Body mass index

CNI: Calcineurin inhibitor

DBP: Diastolic blood pressure

FEV1: Forced expiratory volume during one second

FVC: Forced vital capacity

ICU: Intensive care unit

IQR: Interquartile range

LTx: Lung transplantation

$\mathrm{OH}$ : Orthostatic hypotension

PGD: Primary graft dysfunction

QOL: Quality of life

SBP: Systolic blood pressure.

\section{Data Availability}

The data used to support the findings of this study are available from the corresponding author upon reasonable request.

\section{Conflicts of Interest}

The authors declare that they have no conflicts of interest. 


\section{Authors' Contributions}

All authors have made substantial contributions to the conception or design of the work, acquisition, analysis, or interpretation of data for the work, drafting the work or revising it critically for important intellectual content, and final approval of the version to be published in accordance with ICMJE guidelines. All authors agree to be accountable for all aspects of the work in ensuring that questions related to the accuracy or integrity of any part of the work are appropriately investigated and resolved.

\section{Acknowledgments}

The authors appreciate editorial assistance from Kristine Nally.

\section{References}

[1] H. Kaufmann, "Consensus statement on the definition of orthostatic hypotension, pure autonomic failure and multiple system atrophy," Clinical Autonomic Research: Official Journal of the Clinical Autonomic Research Society, vol. 6, no. 2, pp. 125-126, 1996.

[2] W. Wieling and I. J. Schatz, "The consensus statement on the definition of orthostatic hypotension: a revisit after 13 years," Journal of Hypertension, vol. 27, no. 5, pp. 935-938, 2009.

[3] C. J. Mathias, "Autonomic diseases: clinical features and laboratory evaluation," Journal of Neurology Neurosurgery and Psychiatry, vol. 74, no. 3, p. iii31, 2003.

[4] D. Langer, "Rehabilitation in patients before and after lung transplantation," Respiration, vol. 89, no. 5, pp. 353-362, 2015.

[5] N. Ambrosino, C. Bruschi, G. Callegari et al., "Time course of exercise capacity, skeletal and respiratory muscle performance after heart-lung transplantation," European Respiratory Journal, vol. 9, no. 7, pp. 1508-1514, 1996.

[6] M. N. Bartels, H. F. Armstrong, R. E. Gerardo et al., "Evaluation of pulmonary function and exercise performance by cardiopulmonary exercise testing before and after lung transplantation," Chest, vol. 140, no. 6, pp. 1604-1611, 2011.

[7] A. C. Krieger, P. Szidon, and S. Kesten, "Skeletal muscle dysfunction in lung transplantation," The Journal of Heart and Lung Transplantation, vol. 19, no. 4, pp. 392-400, 2000.

[8] L. C. Lands, A. A. Smountas, G. Mesiano et al., "Maximal exercise capacity and peripheral skeletal muscle function following lung transplantation," The Journal of Heart and Lung Transplantation, vol. 18, no. 2, pp. 113-120, 1999.

[9] L. Myaskovsky, M. A. Dew, M. L. McNulty et al., "Trajectories of change in quality of life in 12-month survivors of lung or heart transplant," American Journal of Transplantation, vol. 6 , no. 8, pp. 1939-1947, 2006.

[10] J. G. Pantoja, F. H. Andrade, D. S. Stokić, A. E. Frost, W. L. Eschenbacher, and M. B. Reid, "Respiratory and limb muscle function in lung allograft recipients," American Journal of Respiratory and Critical Care Medicine, vol. 160, no. 4, pp. 1205-1211, 1999.

[11] M. Schwaiblmair, H. Reichenspurner, C. Müller et al., "Cardiopulmonary exercise testing before and after lung and heart-lung transplantation," American Journal of Respiratory and Critical Care Medicine, vol. 159, no. 4 Pt 1, pp. 1277-1283, 1999.

[12] B. Smeritschnig, P. Jaksch, A. Kocher et al., "Quality of life after lung transplantation: a cross-sectional study," The
Journal of Heart and Lung Transplantation, vol. 24, no. 4, pp. 474-480, 2005.

[13] S. M. Studer, R. D. Levy, K. McNeil, and J. B. Orens, "Lung transplant outcomes: a review of survival, graft function, physiology, health-related quality of life and cost-effectiveness," European Respiratory Journal, vol. 24, no. 4, pp. 674-685, 2004.

[14] X. N. Wang, T. J. Williams, M. J. McKenna et al., "Skeletal muscle oxidative capacity, fiber type, and metabolites after lung transplantation," American Journal of Respiratory and Critical Care Medicine, vol. 160, no. 1, pp. 57-63, 1999.

[15] S. Mathur, T. Janaudis-Ferreira, L. Wickerson et al., "Meeting report: consensus recommendations for a research agenda in exercise in solid organ transplantation," American Journal of Transplantation, vol. 14, no. 10, pp. 2235-2245, 2014.

[16] R. D. Yusen, J. D. Christie, L. B. Edwards et al., "The registry of the international society for heart and lung transplantation: thirtieth adult lung and heart-lung transplant report-2013; focus theme: age," The Journal of Heart and Lung Transplantation, vol. 32, no. 10, pp. 965-978, 2013.

[17] M. Schuurmans, J. Wild, A. Pazhenkottil, I. Inci, M. Kohler, and C. Benden, "Orthostatic hypotension appears to be common among lung transplant recipients," Chest, vol. 145, no. 3, p. 633A, 2014.

[18] J. Dizon, K. Chen, M. Bacchetta et al., "A comparison of atrial arrhythmias after heart or double-lung transplantation at a single center," Journal of the American College of Cardiology, vol. 54, no. 22, pp. 2043-2048, 2009.

[19] J. González-Costello, H. F. Armstrong, U. P. Jorde et al., "Chronotropic incompetence predicts mortality in severe obstructive pulmonary disease," Respiratory Physiology \& Neurobiology, vol. 188, no. 2, pp. 113-118, 2013.

[20] V. Martelli, S. Mathur, L. Wickerson et al., "Impaired cardiac autonomic response in lung transplant patients: a retrospective cohort study," Clinical Transplantation, vol. 33, no. 7, Article ID e13612, 2019.

[21] P. K. Stein, P. Nelson, J. N. Rottman et al., "Heart rate variability reflects severity of COPD in PiZ $\alpha 1$-antitrypsin deficiency," Chest, vol. 113, no. 2, pp. 327-333, 1998.

[22] E. Tobaldini, G. Rodrigues, A. Monti et al., "Sympatho-vagal dysfunction in patients with end-stage lung disease awaiting lung transplantation," Journal of Clinical Medicine, vol. 9, no. 4,2020 .

[23] M. D. O'Connell, G. M. Savva, C. Finucane, R. RomeroOrtuno, C. W. Fan, and R. A. Kenny, "Impairments in hemodynamic responses to orthostasis associated with frailty: results from the irish longitudinal study on ageing (TILDA)," Journal of the American Geriatrics Society, vol. 66, no. 8, pp. 1475-1483, 2018.

[24] R. Varadhan, P. H. M. Chaves, L. A. Lipsitz et al., "Frailty and impaired cardiac autonomic control: new insights from principal components aggregation of traditional heart rate variability indices," The Journals of Gerontology Series A: Biological Sciences and Medical Sciences, vol. 64A, no. 6, pp. 682-687, 2009.

[25] S. E. Kocyigit, P. Soysal, E. A. Bulut, A. E. Aydin, O. Dokuzlar, and A. T. Isik, "What is the relationship between frailty and orthostatic hypotension in older adults?" Journal of Geriatric Cardiology, vol. 16, no. 3, pp. 272-279, 2019.

[26] S. Bhagavati, P. Maccabee, E. Muntean, and N. B. Sumrani, "Chronic sensorimotor polyneuropathy associated with tacrolimus immunosuppression in renal transplant patients: case reports," Transplantation Proceedings, vol. 39, no. 10, pp. 3465-3467, 2007. 
[27] J. Fleischer, D. J. McMahon, W. Hembree, V. Addesso, C. Longcope, and E. Shane, "Serum testosterone levels after cardiac transplantation," Transplantation, vol. 85, no. 6, pp. 834-839, 2008.

[28] S. Nygaard, A. H. Christensen, K. Rolid et al., "Autonomic cardiovascular control changes in recent heart transplant recipients lead to physiological limitations in response to orthostatic challenge and isometric exercise," European Journal of Applied Physiology, vol. 119, no. 10, pp. 2225-2236, 2019.

[29] S. Kuten, A. Gaber, D. Nguyen, E. Graviss, S. Patel, and R. Knight, "Neurogenic orthostatic hypotension: an iatrogenic complication of successful pancreas transplantation? [abstract]," American Journal of Transplantation, vol. 17, no. s3, 2017.

[30] A. Khurana, C. F. McCuskey, and E. G. Slavcheva, "Orthostatic hypotension in kidney pancreas transplant patients and its relation to preexisting autonomic neuropathy," Experimental and Clinical Transplantation: Official Journal of the Middle East Society for Organ Transplantation, vol. 6, no. 2, pp. 127-131, 2008.

[31] S. Munjal, C. Kavasmaneck, I. Sekhon, J. Kolmetz, D. Kim, and A. Ejaz, "Alterations in hemodynamic profile in orthostatic hypotension," The Internet Journal of Internal Medicine, vol. 5, no. 2, 2004. 